\title{
Dexketoprofen vs. Tamsulosin vs. Silodosin vs. Tadalafil as Medical Expulsive Therapy for Distal Ureteral Stones in Men
}

\author{
Metin Gur ${ }^{1}$, Muhammet Bahaettin Ulư ${ }^{1}$, Suleyman Tumer Caliskan ${ }^{1}$, Kemal Ozturk ${ }^{1}$ and Ekrem Akdeniz ${ }^{2}$ \\ ${ }^{1}$ Department of Urology, Gazi Hospital, Samsun, Turkey \\ ${ }^{2}$ Department of Urology, Health Practice and Research Center, University of Health Sciences, Samsun, Turkey
}

\begin{abstract}
Objective: To assess the effectiveness of the use of dexketoprofen, tamsulosin, silodosin, and tadalafil in medical expulsive therapy for distal ureteral stones in male patients.

Study Design: Cohort study.

Place and Duration of Study: Department of Urology, Gazi Hospital, Samsun, Turkey, from March 2020 to March 2021.

Methodology: Adult males satisfying the inclusion criteria were randomly assigned into dexketoprofen (Group 1), tamsulosin (Group 2), silodosin (Group 3), or tadalafil (Group 4) treatment arms. The primary endpoint consisted of the stone expulsion rate at the end of four weeks, while the secondary endpoints were the expulsion rate after two weeks and the occurrence of adverse events. Clinical findings were then compared among the study groups.

Results: Altogether 193 patients, 50 (25.9\%) in group 1, 48 (24.9\%) in group 2, 49 (25.4\%) in group 3, and $46(23.8 \%)$ in group 4 , were enrolled in the study. No significant difference was determined in terms of age, body mass index, stone characteristics, expulsion time, pain episodes, or total analgesic consumption among the four groups. Expulsion rates in the fourth week were $48 \%, 79.2 \%, 81.6 \%$, and $78.3 \%$ in groups $1,2,3$, and 4 , respectively. Stone expulsion rates were significantly greater in groups 2,3 , and 4 compared to group 1 ( $p<0.001$ ), but no significant differences were determined between groups 2,3 , and 4 . No severe adverse effects occurred throughout the study period.

Conclusion: Tamsulosin, silodosin and tadalafil exhibited higher expulsion rates for distal ureteral stones in male patients, although none was significantly superior to the others. All three are safe, efficacious, and well-tolerated, with only very minor side-effects.
\end{abstract}

Key Words: Dexketoprofen, Distal ureteral stones, Medical expulsive therapy, Silodosin, Tadalafil, Tamsulosin.

How to cite this article: Gur M, Ulu MB, Caliskan ST, Ozturk K, Akdeniz E. Dexketoprofen vs. Tamsulosin vs. Silodosin vs. Tadalafil as Medical Expulsive Therapy for Distal Ureteral Stones in Men. J Coll Physicians Surg Pak 2021; 31(08):947-952.

\section{INTRODUCTION}

Medical expulsive therapy (MET) is a non-invasive medical treatment used in ureteral stones aimed at achieving spontaneous stone expulsion through relaxation of the smooth ureteral muscles and reduction of peristaltic activity. ${ }^{1}$ Stone passage is contingent on two principal factors, those involving the stone, and those involving the urinary system. Stone-related factors include the stone size, number, and location within the urinary system. Urinary system-related factors include ureteric spasm, mucosal edema or inflammation, and the ureteric anatomy. ${ }^{2}$ The objective of MET is to achieve spontaneous stone passage through relaxation of the smooth muscles of the ureter and by reducing peristaltic activity.

Correspondence to: Dr. Metin Gur, Department of

Urology, Gazi Hospital, Samsun, Turkey

E-mail: drmetingur55@gmail.com

Received: April 18, 2021; Revised: June 25, 2021;

Accepted: July 09, 2021

DOI: https://doi.org/10.29271/jcpsp.2021.08.947
High densities of the three alpha-1 receptor subtypes (alpha 1a, $1 b$, and $1 d$ ) occur in the distal third of ureteric smooth muscle. Alpha blocker therapy suppresses basal smooth muscle tone, together with peristaltic frequency and amplitude, while preserving tonic propulsive contractions, resulting in decreased intra-ureteric pressure and greater fluid transport. ${ }^{3}$ Use of alpha-1 adrenergic receptor blockers thus facilitates stone passage. Tamsulosin, which exhibits high uroselectivity as well as comparable alpha- 1 a and $1 d$ activity, and silodosin, a more selective alpha-1a adrenergic receptor antagonist, are widely employed in research and are of proven efficacy in MET. ${ }^{4}$

Phosphodiesterase- 5 inhibitors (PDE-5is) act on the smooth muscle nitric oxide/cyclic guanosine monophosphate signalling pathway and produce ureteral relaxation. The lumen of the ureter is thus dilated, allowing stones to pass spontaneously. One direct meta-analysis showed that the PDE-5i tadalafil can effectively treat distal ureteral calculi as MET. ${ }^{5}$ Although tadalafil has been employed to treat sexual dysfunction and lower urinary tractsymptoms, its application in MET forthe treatment of ureteral stones is highly limited. The number of studies 
comparing the efficacy of silodosin and tadalafil is very small. Tamsulosin is the alpha-1 adrenoreceptor antagonist most frequently evaluated for the purpose of MET, and is of proven effectiveness. ${ }^{6}$ Although non-steroid anti-inflammatory drugs (NSAIDs) reduce ureteral spasm and lower intraureteral pressure, their use for MET is controversial. ${ }^{7,8}$ The present study therefore investigated the effect of NSAIDs on MET. Dexketoprofen is a non-steroidal anti-inflammatory agent frequently used in Turkey, and was given to the control group in the present research. The purpose of this study was to compare the safety and effectiveness of the use dexketoprofen, tamsulosin, silodosin, and tadalafil in distal ureteralstones.

\section{METHODOLOGY}

Once Ethics Committee Approval had been obtained, male patients were enrolled from March 2020 to March 2021. CONSORT 2010 statement guidelines were used to design and reportthis trial. ${ }^{9}$

Patients receiving MET due to distal ureteral stone and meeting the inclusion criteria were enrolled in this prospective, randomised monocentric study. Stone length was evaluated from non-contrast computed tomography (CT) images using a digital ruler, the highest value being adopted as the stone size. The localisation of distal ureter was defined as distal to the region where the ureter crosses over the iliac artery. Inclusion criteria were male patients aged 18-55 years, with a single stone 4-9.9 $\mathrm{mm}$ in size in the distal ureter.

Exclusion criteria were patients with two or more unilateral stones or bilateral stones and those aged 56 or more, since the anatomy of the intertrigonal region will change in association with potential benign prostatic hyperplasia. Female patients were also excluded since the anatomy of the intertrigonal regions differs to that in men; and tadalafil is not to be given to female patients. Moreover, excluded were individuals with fever or urinary tract infections, with kidney failure or severe hydronephrosis, with a solitary kidney, a horseshoe kidney, a duplex urinary system, or a history of ureter strictures, and patients using alpha adrenoceptor antagonists or daily tadalafil.

Sample size calculation was based on an equation of repeated measures design (two-factor). A sample size of 191 participants was found to be satisfactory for detecting an effect size of 0.3 , with a power of $95 \%$, with four groups and two measurements using $\mathrm{G} *$ power software version 3.1 (Franz Faul, Christian-Albrechts-Universität Kiel, Kiel, Germany). The estimated lost to follow-up rate was $10 \%$. Two hundred and twelve patients were included in the study. The risks and benefits of MET were set out in detail to all the participants. Once written informed consent had been given, the patients were prospectively randomly assigned into four groups. Two hundred and twenty-six patients met the inclusion criteria during the time of the research. However, 14 were subsequently excluded on account of unwillingness to participate, and 19 due to being lost to follow-up (Figure 1).

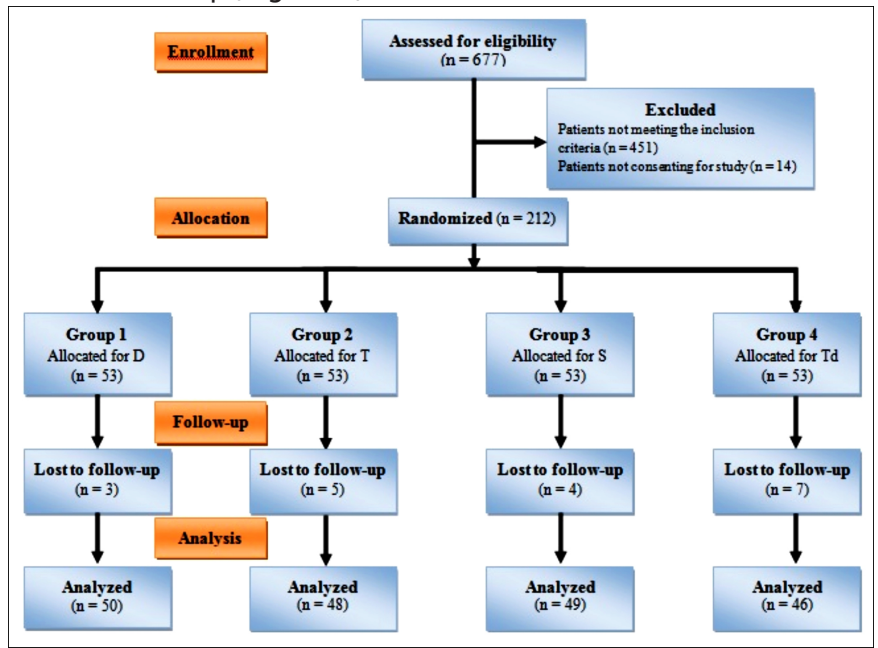

Figure 1: CONSORT flowchart of study design.

(D: Dexketoprofen; T: Tamsulosin; S: Silodosin; Td: Tadalafil).

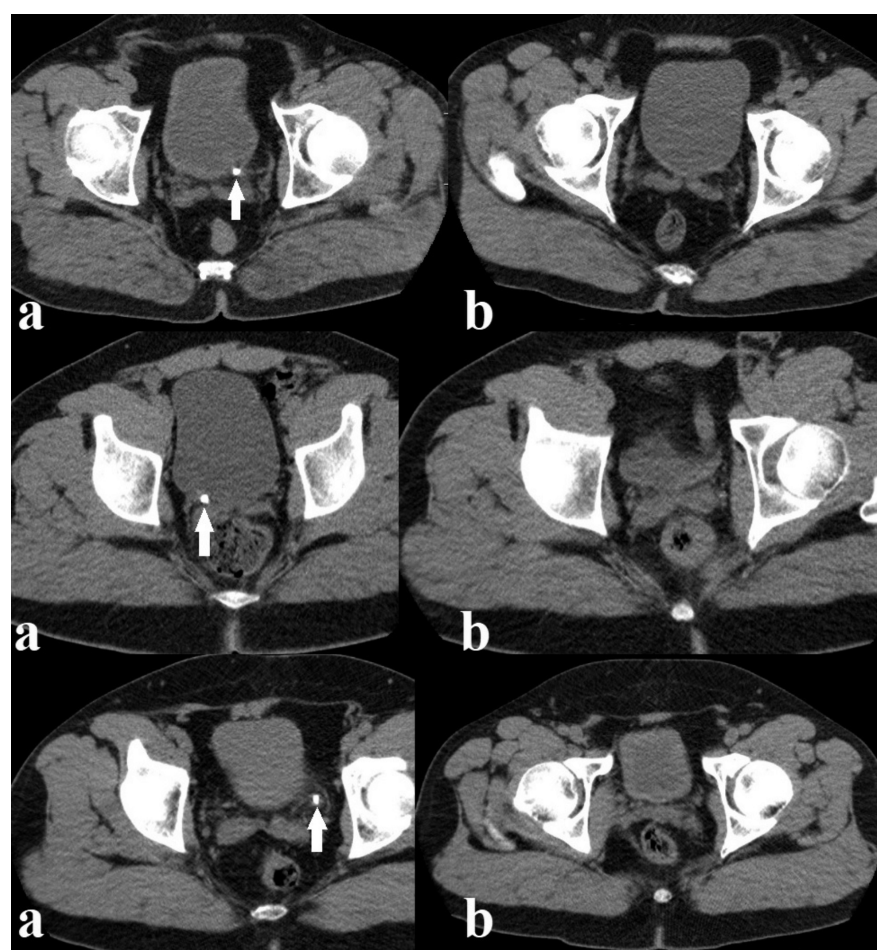

Figure 2: Computed tomography images of patients. (a) Before treatment, (b) Aftertreatment (Arrow: Stone).

The patients were assigned into one of four groups; Group 1 receiving dexketoprofen $50 \mathrm{mg}$, Group 2 receiving tamsulosin $0.4 \mathrm{mg}$, Group 3 receiving silodosin $8 \mathrm{mg}$, and Group 4 receiving tadalafil $5 \mathrm{mg}$ daily. All participants were requested to drink two liters of water every day and to use a sieve for stone collection after urine filtration. Patients were also permitted to use $1 \mathrm{~g}$ intramuscular (IM) metamizole for analgesia on demand. Patients were advised to present to hospital in case of intractable acute pain, fever, urinary tract infection, or nausea and vomiting. 
Table I: Patients' characteristics and the results of the study values are presented as median (interquartile range) or number ( $\mathrm{n}, \%$ ).

\begin{tabular}{|c|c|c|c|c|c|}
\hline Variables & $\begin{array}{l}\text { Group } 1 \\
(n=50)\end{array}$ & $\begin{array}{l}\text { Group } 2 \\
(n=48)\end{array}$ & $\begin{array}{l}\text { Group } 3 \\
(n=49)\end{array}$ & $\begin{array}{l}\text { Group } 4 \\
(n=46)\end{array}$ & $\mathbf{p}$ \\
\hline Age (years) & $38(31.5-48)$ & $41(30.25-51.75)$ & $41(33-50.5)$ & $39(31.75-48)$ & 0.814 \\
\hline Body mass index $\left(\mathrm{kg} / \mathrm{m}^{2}\right)$ & $25.94(24.86-28.44)$ & $27.65(25-29.34)$ & $27.15(25.3-28.8)$ & $28.13(24.6-30.7)$ & 0.396 \\
\hline $\begin{array}{l}\text { Side, N (\%) } \\
\text { Right } \\
\text { Left }\end{array}$ & $\begin{array}{l}26(52 \%) \\
24(48 \%)\end{array}$ & $\begin{array}{l}24(50 \%) \\
24(50 \%)\end{array}$ & $\begin{array}{l}24(49 \%) \\
25(51 \%)\end{array}$ & $\begin{array}{l}22(47.8 \%) \\
24(52.2 \%)\end{array}$ & 0.980 \\
\hline $\begin{array}{l}\text { Maximum stone size (mm) } \\
\text { Expulsion rate (after two weeks) } \\
\text { Expulsion rate (after four weeks) }\end{array}$ & $\begin{array}{c}5.75(4.77-8.12) \\
42 \%(21) \\
48 \%(24)\end{array}$ & $\begin{array}{l}6.2(4.62-7.67) \\
68.8 \%(33) \\
79.2 \%(38) \\
\end{array}$ & $\begin{array}{l}6.2(5.1-7.55) \\
71.4 \%(35) \\
81.6 \%(40) \\
\end{array}$ & $\begin{array}{l}6.1(5.17-7.37) \\
65.2 \%(30) \\
78.3 \%(36) \\
\end{array}$ & $\begin{array}{r}0.798 \\
0.010 \\
<0.001 \\
\end{array}$ \\
\hline Expulsion time (day) & $11(8-19.25)$ & $11(7-16.5)$ & $9(7-15)$ & $11(7-15.25)$ & 0.444 \\
\hline Pain episodes & $1(0-2)$ & $1(0-1)$ & $0(0-1)$ & $0(0-1)$ & 0.685 \\
\hline Average dosage of metamizol (g) & $1(0-2)$ & $1(0-1)$ & $0(0-1)$ & $0(0-2)$ & 0.862 \\
\hline
\end{tabular}

Table II: Post-hoc analyses for expulsion proportions in the study.

\begin{tabular}{|l|c|c|}
\hline \multirow{2}{*}{} & Week 2 & Week 4 \\
\cline { 2 - 3 } & $\mathbf{p}$ & $\mathbf{p}$ \\
\hline Group 1 vs. Group 2 & 0.008 & 0.001 \\
\hline Group 1 vs. Group 3 & 0.002 & $<0.001$ \\
\hline Group 1 vs. Group 4 & 0.023 & 0.002 \\
\hline Group 2 vs. Group 3 & 0.773 & 0.760 \\
\hline Group 2 vs. Group 4 & 0.716 & 0.915 \\
\hline Group 3 vs. Group 4 & 0.515 & 0.681 \\
\hline
\end{tabular}

Patients' clinical conditions were monitored by means of weekly telephone interviews. These were conducted by a research fellow, who investigated whether or not the stone had passed, the patient's clinical state, and development of any drug-related side-effects. MET was discontinued in case of severe pain despite IM metamizole, adverse events, severe hydronephrosis, kidney failure, fever, or urinary tract infection, if the patient expressed a desire for stone removal, and ureteroscopic lithotripsy (UL) was performed. Patients who reported expelling their stones underwent CT to confirm their stone-free status (Figure 2). Clinically stable patients, who were unable to expel their stones or who were uncertain whether or not they had done so, were invited to attend the outpatient clinic on days 14 and 28. Stone and hydronephrosis status were assessed using CT. Patients also underwent routine urine culture, complete urine, and kidney function tests. UL was recommended to patients who were unable to expel their stones at the end of 28-day follow-up.

Cumulative stone passage rates over a four-week treatment period represented the primary endpoint. The secondary end-points were expulsion rates after two weeks of treatment, time to stone passage, numbers of colic episodes, analgesic doses, and side-effects. Time to stone passage was defined as the period elapsed between the date of enrollment to confirmation of stone passage, or to the follow-up visit when the presence of any visible stone was no longer revealed by imaging studies.

SPSS 25 (Statistical Package for Social Sciences- IBM Corp.,
Armonk, NY, USA) software was employed for data analysis. Normality of measurable data was assessed using the Shapiro-Wilk tests. Non-normally distributed continuous data as median (interquartile range [IQR]: $25^{\text {th }}$ percentile- $75^{\text {th }}$ percentile). The Kruskal-Wallis test was applied to assess statistically significant differences among the groups. Nominal variables were expressed as numbers and percentages and analyzed using the chi-square test and the Fisher-Freeman Halton Exact test. A p-values $<0.05$ were considered statistically significant. The Bonferroni post-hoc test was applied to determine the differences between pairwise groups in order to identify the source of significance in variables identified as significant.

\section{RESULTS}

No statistically significant differences were observed between the groups in terms of patient body mass index, age, stone side, stone size, expulsion time, pain episodes, or average dosage of metamizole. Patient characteristics and outcomes are presented in Table I.

Two-week stone expulsion rates were $42 \%$ ( 21 out of 50 patients) in Group 1, 68.8\% (33 out of 48 ) in Group 2, 71.4\% (35 out of 49) in Group 3, and 65.2\% (30 out of 46) in Group 4. Intergroup differences found to be statistically significant $(p=0.010)$. While the efficacy of silodosin was significantly superior to that of dexketoprofen $(p=0.002)$, no difference emerged among the other study groups.

Four-week stone expulsion rates were $48 \%$ (24 out of 50 patients) in Group 1, 79.2\% (38 out of 48) in Group 2, 81.6\% ( 40 out of 49 ) in Group 3, and $78.3 \%$ (36 out of 46 ) in Group 4. Statistically significant differences were determined between the groups $(p<0.001)$. The stone expulsion rate after four weeks differed significantly between Group 1 and Group 2, Group 1 and Group 3, and Group 1 and Group 3. Groups 2, 3 and 4 were superior to Group 1 in terms of stone expulsion after four weeks, although no significant difference in terms of efficacy was observed between groups 2, 3 and 4. Post-hoc analyses for expulsion rates in the study and the results obtained are given in Table II. 
Table III: Adverse events in each group.

\begin{tabular}{|c|c|c|c|c|c|}
\hline Variable, N (\%) & Group $1(n=50)$ & Group $2(n=48)$ & Group $3(n=49)$ & Group $4(n=46)$ & $\mathbf{p}$ \\
\hline Abnormal ejaculation & 0 & $4(8.3 \%)$ & $8(16.3 \%)$ & $2(4.3 \%)$ & 0.009 \\
\hline Headache & 0 & $5(10.4 \%)$ & $6(12.2 \%)$ & $7(15.2 \%)$ & 0.019 \\
\hline Orthostatic hypotension & $2(4 \%)$ & $6(12.5 \%)$ & $3(6.1 \%)$ & $3(6.5 \%)$ & 0.487 \\
\hline Backache & 0 & $4(8.3 \%)$ & $5(10.2 \%)$ & $7(15.2 \%)$ & 0.023 \\
\hline Gastritis & $15(30 \%)$ & $3(6.3 \%)$ & $2(4.1 \%)$ & $3(6.5 \%)$ & $<0.001$ \\
\hline Total & $17(34 \%)$ & $22(45.8 \%)$ & $24(49 \%)$ & $22(47.8 \%)$ & 0.414 \\
\hline
\end{tabular}

No severe drug-associated adverse effects were recorded in any of the study groups. No intergroup difference was also determined in complication rates $(p=0.414)$. Headache and backache were more frequent in Group 4, abnormal ejaculation was more frequent in Group 3, and gastritis was significantly more common in Group 1 ( $p<0.05)$. The prevalences of backache $(15.2 \%)$ and headache $(15.2 \%)$ in the tadalafil group in the present study were significantly higher than in the control group. However, there was no significant difference between groups, 2, 3, and 4 in terms of backache or headache. The prevalences of abnormal ejaculation in the silodosin group in the present study were significantly higher than in the other groups. Greater orthostatic hypotension was reported in all groups, and was more frequent in Group 2 , although the difference was not statistically significant ( $p$ $=0.487$ ). Adverse effects are shown in Table III.

\section{DISCUSSION}

The American Urological Association (AUA) and European Association of Urology (EAU) clinical guidelines support the use of MET for distal ureteral calculi. However, neither guideline sets out the ideal stone size for MET, nor the ideal length of treatment. ${ }^{6,10,11}$ One study reported wide variation in observed spontaneous passage rates, between $71 \%$ and $98 \%$ for distal ureteral stones less than $5 \mathrm{~mm}$ in size, and between $25 \%$ and $53 \%$ for stones $5-10 \mathrm{~mm}$ in size, with a mean expulsion time exceeding 10 days. ${ }^{12}$ The AUA states that spontaneous passage rates increase as the stone size decreases, and that MET may be suitable for distal stones with a size of $10 \mathrm{~mm} .{ }^{6}$ However, the EAU emphasises the possibility of differences among patients, and that it is not possible to estimate an exact cut-off size for stones with a high likelihood of being passed spontaneously, although it cites a best approximation of less than $10 \mathrm{~mm}$. Small stones (less than $6 \mathrm{~mm}$ ) have also been described as ideal for MET. ${ }^{11}$

There is no specific information available for the duration of MET, although many authors have reported a follow-up time of four weeks. ${ }^{5,13}$ The AUA recommends that observation of patients with complete unilateral ureteral obstruction should not exceed six weeks in order to avoid irreversible kidney damage. ${ }^{6}$ As with the typical size of stone, the EAU also gives no specific follow-up time, merely reporting that informed patients not developing any complications (such as infection, refractory pain, or deterioration of renal function) may be placed under observation. ${ }^{11}$
Numerous medical therapies have been investigated in the context of MET, including antispasmodics, corticosteroids, alpha blockers, calcium channel blockers, PDE-5is, and combinations thereof. ${ }^{8}$ Alpha blockers are currently the only monotherapy recommended for use as MET by the EAU. ${ }^{11}$ Significantly higher success rates have been reported in patients using alpha blockers in the treatment of distal ureteral stones less than $10 \mathrm{~mm}$ in size compared to patients receiving placebo or no treatment ( 77.3 vs. $54.4 \%$, respectively). ${ }^{6}$ A meta-analysis of alpha blockers compared to controls determined a statistically significant absolute increase of $29 \%$ in stone-passage rates. ${ }^{10} \mathrm{PDE}-5 \mathrm{i}$ is have recently frequently been employed for MET, and one meta-analysis described tadalafil and sildenafil as markedly superior to placebo. ${ }^{8}$ The success rate for tadalafil in distal ureteral stones smaller than $10 \mathrm{~mm}$ ranges between $66.7 \%$ and $84.1 \%{ }^{14-17}$

Analysis of stone expulsion rates after four weeks, the first endpoint of this study in groups $1,2,3$, and 4 revealed $48 \%$, $79.2 \%, 81.6 \%$, and $78.3 \%$ efficacy, respectively. The success rates of tamsulosin, silodosin and tadalafil were significantly higher compared to the dexketoprofen group. All three agents exhibited comparable efficacy among themselves.

Randomised controlled studies involving the use of tamsulosin and silodosin in the treatment of distal ureteral stones smaller than $10 \mathrm{~mm}$ have reported significantly high success rates of $57-64.4 \%$ and $80.3-84 \%$, respectively. However, the success rate for tamsulosin in those studies is relatively lower than that in other studies. ${ }^{17-19}$ In contrast, Ye et al.'s randomised controlled study of 3,296 patients $(1,642$ in the tamsulosin group and 1,654 in the placebo group) yielded a success rate for tamsulosin of $86 \% .{ }^{13}$ In their meta-analysis, Tao et al. reported a success rate for tamsulosin in distal ureteral stones of $67-90.7 \% .{ }^{20}$ In terms of tamsulosin success rates, the present study is consistent with Ye et al. and Tao et al.

Some studies involving male and female patients have determined significantly higher success rates for silodosin compared to tamsulosin ${ }^{17-19}$, but not others. ${ }^{21,22}$ Success rates for tamsulosin and silodosin in the present study were $79.2 \%$ and $81.6 \%$, respectively, the difference being statistically insignificant. The equivalent rates were $82 \%$ and $88 \%$ in Imperatore et al., and $72.4 \%$ and $78.6 \%$ in Arda et al. studies, and similarly to the present research; both these studies found no significant difference between tamsulosin 
and silodosin. ${ }^{21,22}$ However, men and women were enrolled in both those studies, while this study involved only young/middle-aged men. This represents the major difference between those studies and this research.

The stone expulsion rate for tadalafil in the fourth week exceeded $78.3 \%$. Randomised controlled studies in the literature have reported success rates for tadalafil between $66.7 \%$ and $84.1 \% .{ }^{14-17}$ Success rates for tadalafil were significantly higher than those for tamsulosin in two of those studies, ${ }^{15,16}$ but not in two others. ${ }^{14,17}$ The only randomised controlled study in the literature comparing tadalafil monotherapy and silodosin therapy reported success rates of $83.3 \%$ for silodosin and $66.7 \%$ for tadalafil, the difference being significant $(p=0.016) .{ }^{17}$ The effectiveness of tadalafil was found to be similar to those of silodosin and tamsulosin in the present study.

Stone expulsion rates after two weeks in groups 1, 2, 3, and 4 indicated $42 \%, 68.8 \%, 71.4 \%$ and $65.2 \%$ efficacy, respectively $(p=0.010)$. Similarly to the present study, Dell'Atti reported success rates for tamsulosin and silodosin at the end of two weeks of $43.2 \%$ and $69.69 \%$, while Arda et al. reported rates of $58.3 \%$ and $62.3 \%$, respectively. ${ }^{19,22}$

One previous study reported that antispasmodics, watchful waiting, and placebo yielded low rates of stone passage, while higher rates were achieved with alpha blockers and PDI-5is. ${ }^{8}$ Group 1 in the present study was given dexketoprofen, which is frequently used in our county, with a success rate of $48 \%$. Arda et al. reported $50 \%$ success for watchful waiting. ${ }^{22}$ The AUA has reported a success rate of $54.4 \%$ for placebo or no treatment. ${ }^{6}$ The effectiveness of dexketoprofen for MET in the present study was lower than that of placebo or no treatment. However, both men and women were enrolled in both those studies, while this study involved only young/middle-aged men. The authors attribute the poorer performance of dexketoprofen compared to placebo or no treatment to no specific patient group being enrolled in this study.

The treatment groups in the present study exhibited no significant differences in terms of expulsion time, pain episodes, or total analgesic consumption. In contrast to this study, a randomised, controlled study involving male and female patients and comparing tamsulosin, silodosin and tadalafil monotherapies found that silodosin was superior to tamsulosin and tadalafil in terms of expulsion time, pain episodes, and total analgesic use. ${ }^{17}$ Similarly to the present research, another previous study comparing tadalafil and tamsulosin also reported no difference in expulsion time, pain episodes, or total analgesic consumption. ${ }^{16}$

In addition to the endpoints of this study, no difference was observed in terms of complication rates $(p=0.414)$. When the drugs were compared in terms of common side-effects, significant differences were observed in terms of headache, backache, abnormal ejaculation and gastritis, but no difference was determined in orthostatic hypotension. Consistent with previous studies, retrograde ejaculation, a more specific alpha blocker side-effect, was more common in patients using silodosin. ${ }^{17-19,21}$ The prevalences of backache (15.2\%) and headache (15.2\%) in the tadalafil group in the present study were significantly higher than in the control group. However, there was no significant difference between groups 2, 3, and 4 in terms of backache or headache. Consistent with the present research, other studies comparing tadalafil and tamsulosin have also observed no significant intergroup differences in terms of backache or headache. ${ }^{14-17}$ From that perspective, this study is compatible with the previous literature.

\section{CONCLUSION}

MET seems to be a particularly effective method for treating distal ureteral stones between $4 \mathrm{~mm}$ and $9.9 \mathrm{~mm}$ in size in adult males. The results of the present study suggested no significant superiority in terms of effectiveness or duration between tamsulosin, silodosin and tadalafil for distal ureteral stones, with minimal drug side-effects. The effectiveness of dexketoprofen for the purpose of MET was similar to that of placebo or no treatment.

\section{ETHICAL APPROVAL:}

All procedures involving human participants were conducted in strict compliance with the ethical principles of the Institutional Research Committee and of the 1964 Declaration of Helsinki and subsequent modifications or equivalent ethical standards. This study was approved by Health Sciences University, Medical Ethics Committee (Ref. No. GOKA/2020/16/4 Date: 09.02.2020).

\section{PATIENTS' CONSENTS:}

Informed consents were obtained from all subjects on enrolment.

\section{CONFLICT OF INTEREST:}

The authors declared no conflict of interest.

\section{AUTHORS' CONTRIBUTION:}

MG: Conception of the work, acquisition of data, drafting the work, and final approval.

MBU: Acquisition of data, literature search, drafting the work, and final approval.

STC: Design of the work, acquisition of data, drafting the work, and final approval.

KO: Conception of the work, acquisition of data, drafting the work, and final approval.

EA: Design of the work, analysis of data, literature search, drafting the work, and final approval.

\section{REFERENCES}

1. Somani BK, Aboumarzouk O, Traxer O, Baard J, Kamphuis G, de la Rosette J. Medical expulsive therapy for ureteral 
stones: Where do we go from here? Nat Rev Urol 2016; 13(10):608-12. doi: 10.1038/nrurol.2016.146.

2. Ibrahim Al, Shetty SD, Awad RM, Patel KP. Prognostic factors in the conservative treatment of ureteric stones. Br J Urol 1991; 67(4):358-61. doi: 10.1111/j.1464-410x.1991.tb15161.x.

3. Yilmaz E, Batislam E, Basar MM, Tuglu D, Ferhat M, Basar H. The comparison and efficacy of 3 different alpha1-adrenergic blockers for distal ureteral stones. J Urol 2005; 173(6):2010-2. doi: 10.1097/01.ju.0000158453.60029.0a.

4. Campschroer T, Zhu X, Vernooij RW, Lock MT. Alphablockers as medical expulsive therapy for ureteral stones. Cochrane Database Syst Rev 2018; 4(4):CD008509. doi: 10.1002/14651858.CD008509.pub2.

5. Liu Z, Su J, Yuan D, Zhang Y, Wang W, Jiao K, et al. Efficacy and safety of PDE5-Is and $\alpha-1$ blockers for treating distal ureteral calculi: A mixed treatment comparison network meta-analysis of randomised controlled clinical trials. Int J Clin Exp Med 2019; 12(5):4623-37.

6. Assimos D, Krambeck A, Miller NL, Monga M, Murad MH, Nelson CP, et al. Surgical Management of Stones: American Urological Association/Endourological Society Guideline, PART II. J Urol 2016; 196(4):1161-9. doi: 10.1016/ j.juro.2016.05.091.

7. Holmlund D. On medical treatment for ureteral stone expulsion. Scand J Urol 2018; 52(2):94-100. doi: 10.1080/21681805.2018.1428682.

8. Liu H, Wang S, Zhu W, Lu J, Wang X, Yang W. Comparative efficacy of 22 drug interventions as medical expulsive therapy for ureteral stones: A systematic review and network meta-analysis. Urolithiasis 2020; 48(5):447-57. doi: 10.1007/s00240-019-01159-5.

9. Moher D, Hopewell S, Schulz KF, Montori V, Gøtzsche PC, Devereaux PJ, et al. CONSORT 2010 explanation and elaboration: Updated guidelines for reporting parallel group randomised trials. BMJ 2010; 340:c869. doi: 10.1136/bmj.c869.

10. Preminger GM, Tiselius HG, Assimos DG, Alken P, Buck C, Gallucci $M$, et al. 2007 guideline for the management of ureteral calculi. J Urol 2007; 178(6):2418-34. doi: 10.1016/ j.juro.2007.09.107.

11. Türk C, Neisius A, Petrik A, Seitz C, Skolarikos A, Thomas K, et al. EAU Guidelines of Urolithiasis. Arnhem: EAU; 2020 [cited 2021 Feb 26]. Available from: http://uroweb.org/guideline/urolithiasis/\#3.

12. Bensalah K, Pearle M, Lotan Y. Cost effectiveness of medical expulsive therapy using alpha-blockers for the treatment of distal ureteral stones. Eur Urol 2008; 53(2):411-8. doi: 10.1016/j.eururo.2007.09.012.

13. Ye Z, Zeng G, Yang H, Tang K, Zhang X, Li H, et al. Efficacy and safety of tamsulosin in medical expulsive therapy for distal ureteral stones with renal colic: A multicenter, randomised, double-blind, placebo-controlled trial. Eur Urol 2018; 73(3):385-391. doi: 10.1016/j.eururo.2017.10.033.

14. Goyal SK, Singh V, Pandey H, Chhabra MK, Aggarwal SP, Bhat A. Comparative efficacy of tamsulosin versus tadalafil as medical expulsive therapy for distal ureteric stones. Urol Ann 2018; 10(1):82-6. doi: 10.4103/UA.UA_36_17.

15. Puvvada S, Mylarappa P, Aggarwal K, Patil A, Joshi P, Desigowda R. Comparative efficacy of tadalafil versus tamsulosin as the medical expulsive therapy in lower ureteric stone: A prospective randomised trial. Cent European J Urol 2016; 69(2):178-82. doi: 10.5173/ceju.2016.766.

16. Kc HB, Shrestha A, Acharya GB, Basnet RB, Shah AK, Shrestha PM. Tamsulosin versus tadalafil as a medical expulsive therapy for distal ureteral stones: A prospective randomized study. Investig Clin Urol 2016; 57(5):351-6. doi: 10.4111/icu.2016.57.5.351.

17. Kumar S, Jayant K, Agrawal MM, Singh SK, Agrawal S, Parmar KM. Role of tamsulosin, tadalafil, and silodosin as the medical expulsive therapy in lower ureteric stone: A randomised trial. Urology 2015, 85:59-63. doi: 10.1016/ j.urology.2014.09.022.

18. Gupta S, Lodh B, Kaku SA, Somarendra K, Sholay MK, Rajendra SS. Comparing the efficacy of tamsulosin and silodosin in the medical expulsion therapy for ureteral calculi. J Clin Diagn Res 2013, 7:1672-4. doi: 10.7860/JCDR/ 2013/6141.3241.

19. Dell'Atti L. Silodosin versus tamsulosin as medical expulsive therapy for distal ureteralstones: A prospective randomised study. Urologia 2015; 82(1):54-7. doi: 10.5301/uro. 5000083.

20. Tao RZ, Qin ZQ, Liu FD, Lv JL. Efficacy and safety of tamsulosin in the medical expulsion therapy for distal ureteral calculi: A systematic review and meta-analysis of placebo-controlled trials. Urol J 2019; 16(3):224-31. doi: 10.22037/uj.v0i0.4758.

21. Imperatore V, Fusco F, Creta M, Di Meo S, Buonopane R, Longo $\mathrm{N}$, et al. Medical expulsive therapy for distal ureteric stones: tamsulosin versus silodosin. Arch Ital Urol Androl 2014; 86(2):103-7. doi: 10.4081/aiua.2014.2.103.

22. Arda E, Cakiroglu B, Yuksel I, Akdeniz E, Cetin G. Medical expulsive therapy for distal ureteral stones: Tamsulosin versus silodosin in the Turkish population. Cureus 2017; 9(11):e1848. doi: 10.7759/cureus.1848. 